\title{
Computer Simulation of Transition Regimes of Solitons in Stimulated Raman Scattering with Excitation of Polar Optical Phonons
}

\author{
Galyna Feshchenko, Vladimir Feshchenko \\ ${ }^{1}$ Vanier College, Montreal, Canada \\ ${ }^{2}$ Dawson College, Montreal, Canada \\ Email: feshcheg@vaniercollege.qc.ca, vfeshchenko@place.dawsoncollege.qc.ca
}

Received 7 August 2015; accepted 7 September 2015; published 11 September 2015

Copyright (C) 2015 by authors and Scientific Research Publishing Inc.

This work is licensed under the Creative Commons Attribution International License (CC BY). http://creativecommons.org/licenses/by/4.0/

\begin{abstract}
The system of nonlinear equations modeling the process of nonstationary stimulated Raman scattering (SRS) in noncentrosymmetric crystals for the waves on laser, Stokes, polariton, and phonon frequencies is investigated by using the numerical methods. The general case for amplitudes of waves that resulted in doubling of the number of equations is considered. It is shown that the application of the methods of finite differences to the computer simulation of transition regimes is completely consistent with the analytical results found for the asymptotical solutions in form of solitons. The obtained results also indicate that the laser pulses of Gaussian shape appearing at the boundary of nonlinear medium tend to become solitons of Lorentzian shape. It was also found that the formation of solitons occurs when the vibrations of optical phonons and that of electromagnetic wave were either in or out of phase. It is shown that all electromagnetic waves entering the medium with different speeds become solitons having the same speed. In the second part of the paper we considered the computer simulation of soliton stability with respect to small (weak) perturbations of all interacting waves. In the present paper we considered the case of evolution of those disturbances in the vicinity of peaks of solitons. The numerical analysis showed that in wide range of parameters the solitons were stable.
\end{abstract}

\section{Keywords}

Finite Differences Method, Abbreviated Maxwell's Equations, Stimulated Raman Scattering, Stability 


\section{Introduction}

A fast progress in producing ultrashort pulses stimulates the constantly growing interest in obtaining the additional information on both the optical characteristics of matter (the longitudinal and transverse relaxation times, the constants of the transition, etc.) and the possibility of creation of super short pulses propagating in a medium without energy losses [1] [2]. One of the effects that results in solitons is SRS under the conditions of the interaction of ultrashort pulses of exciting and Stokes radiation in a medium [3]-[5]. The effect of SRS is modeled by the system of abbreviated Maxwell's equations for the amplitudes of the electromagnetic fields and the equations of motion for the averaged polarization [6]. In many cases due to the complexity only the asymptotic behavior of those systems can be found [7]. However, to make clear, whether the SRS pulses go over to the soliton regime, it is necessary to solve the complete set of nonstationary equations which describes the temporal evolution of all interacting waves from the boundary of the medium. It is also important to consider the stability of the SRS solitons [8]. Using different numerical methods in computer simulation of space-time evolution of the interacting waves is the effective approach in analysis of the equations analytical solutions of which could not be found [9]-[11]. In this paper we use the assumption that solitons can exist if, at least, three conditions are satisfied: 1) the asymptotical solution in form of solitons is found; 2) the results of transition regime are consistent with the ones found in the previous part; 3 ) the obtained results are stable with respect to small (weak) perturbations. In our previous paper we established the possibility of existence of the solitons in nonstationary SRS with excitation of polar optical phonons [12]. In the present work it has been shown that the results of the computer simulation of the system of nonlinear equations (see [12]) are consistent with the asymptotic solutions. The computer simulation was provided in Sections 3 and 4. The stability of soliton propagation was studied in Section 5.

\section{Basic Equations}

Let us assume that the pump laser wave with frequency $\omega_{l}$ and Stokes wave $\omega_{s}$ excite both the polariton radiation $\omega_{p}=\omega_{l}-\omega_{s}$ and the polar optical phonon $\omega_{f}$, in the vicinity of which falls $\omega_{p}$ ( the vibration $\omega_{f}$ is supposed to be dipole-active and solitary). The two optical impulses with $\omega_{l, s}$ propagate in noncentrosymmetric crystal at the angles $\theta_{l, s}$ with respect to z-axis (perpendicular to the crystal plane). The vibration $\omega_{f}$, due to its combinational activity, is excited by the cubic nonlinear polarization and electric field on $\omega_{p}$. The electric strengths are:

$$
\begin{gathered}
\boldsymbol{E}_{l, s}(\boldsymbol{r}, t)=\hat{e}_{l, s} A_{l, s}(z, t) \exp \left[i\left(\boldsymbol{k}_{l, s} \boldsymbol{r}-\omega_{l, s} t\right)\right]+c . c ., \\
\boldsymbol{E}_{p}(\boldsymbol{r}, t)=\hat{e}_{p} A_{p}(z, t) \exp \left[i\left(\boldsymbol{W r}-\omega_{p} t\right)\right]+c . c .,
\end{gathered}
$$

where: $\boldsymbol{W}=\boldsymbol{k}_{l}-\boldsymbol{k}_{s}, \quad \boldsymbol{k}_{l, s}$ are the wave vectors in linear theory; $\hat{e}_{l, s, p}$ are the unit vectors ( we assume that only waves of certain fixed polarization are effectively involved into interaction); $A_{t, s, p}(z, t)$ are the amplitudes of interacting waves. The phonon vibration is:

$$
\boldsymbol{Q}_{f}(\boldsymbol{r}, t)=\hat{e}_{f} u_{f}(z, t) \exp \left[i\left(\boldsymbol{W r}-\omega_{f} t\right)\right]+\text { c.c. },
$$

where $\hat{e}_{f}$ is collinear with $\hat{e}_{i p}$. We neglect the pumping of energy into the anti-Stokes and higher Stokes components, since we consider the case in which the intense fields of the exciting (laser) and first Stokes waves are present at the input of the medium. The basic system of nonlinear equations was found in [7] (the first three equations are abbreviated Maxwell's whereas the fourth one is the equation that simulates the behavior of the nonlinear medium):

$$
\begin{gathered}
\frac{\partial A_{l}^{*}}{\partial z}+\frac{1}{v_{l}^{z}} \frac{\partial A_{l}^{*}}{\partial t}=-i \frac{2 \pi q_{l}^{2}}{k_{l}^{z}}\left(\chi_{0} A_{s}^{*} A_{p}^{*}+\frac{\eta_{f} \omega_{f}^{2} \alpha^{(f)}}{g^{(f)}} A_{s}^{*} \tilde{u}_{f}^{*}+\gamma_{0}\left(\left|A_{l}\right|^{2}+\left|A_{s}\right|^{2}\right) A_{l}^{*}\right), \\
\frac{\partial A_{s}^{*}}{\partial z}+\frac{1}{v_{s}^{z}} \frac{\partial A_{s}^{*}}{\partial t}=-i \frac{2 \pi q_{s}^{2}}{k_{s}^{z}}\left(\chi_{0} A_{l}^{*} A_{p}+\frac{\eta_{f} \omega_{f}^{2} \alpha^{(f)}}{g^{(f)}} A_{l}^{*} \tilde{u}_{f}+\gamma_{0}\left(\left|A_{l}\right|^{2}+\left|A_{s}\right|^{2}\right) A_{s}^{*}\right), \\
\frac{\partial A_{p}^{*}}{\partial z}+\frac{1}{v_{p}^{z}} \frac{\partial A_{p}^{*}}{\partial t}=i \frac{q_{p}^{2} \varepsilon_{p}^{\infty}}{2 w^{z}}\left(\frac{w^{2}}{q_{p}^{2} \varepsilon_{p}^{\infty}}-1\right) A_{p}^{*}-i \frac{s_{f} \omega_{f}^{2} \omega_{p}^{2}}{2 g^{(f)} w^{z} c^{2}} \tilde{u}_{f}^{*}-i \frac{2 \pi q_{p}^{2}}{w^{z}} \chi_{0} A_{l}^{*} A_{s},
\end{gathered}
$$




$$
\frac{\partial \tilde{u}_{f}^{*}}{\partial t}+\frac{\gamma_{f}}{2} \tilde{u}_{f}^{*}=i \Delta \omega \tilde{u}_{f}^{*}-i \frac{g^{(f)}}{2 \omega_{f}} A_{p}^{*}-\frac{2 \pi \eta_{f} g^{(f)} \alpha^{(f)}}{s_{f} \omega_{f}} A_{1}^{*} A_{s},
$$

where: $\chi_{0}$ and $\gamma_{0}$ are the corresponding tensor contractions of nonresonance quadratic and cubic nonlinear polarizabilities with unit vectors of polarization of interacting waves; $\eta_{f}=\left(s_{f} /\left(h \omega_{f}\right)\right)^{1 / 2} ; s_{f}$ is the oscillator strength of the oscillation $\omega_{f} ; g^{(f)}$ is the ratio of effective charge to effective mass for given vibration; $\varepsilon_{p}^{\infty}$ is the nonresonance part of dielectric permeability on frequency $\omega_{p} ; q_{l, s, p}=\omega_{l, s, p} / c ; \alpha^{(f)}=e_{s}^{i} e_{l}^{j} \alpha_{i j}^{(f)} ; \alpha_{i j}^{(f)}$ is the tensor of combinational scattering per one elementary cell ; $\tilde{u}_{f}=u_{f} \cdot \exp (-i \Delta \omega t) ; \Delta \omega=\omega_{f}-\omega_{p} ; \gamma_{f}$ is constant of phonon attenuation; $v_{l, s, p}^{z}$-z-components of velocities of waves on $\omega_{l, s, p}$.

The asymptotic solution of the system (4)-(7) in form of solitons was found in [12]. Soliton amplitudes $B_{l, s, p, f}(\xi)$ and phases $\Phi_{l, s . p . f}(\xi)$ were related with $A_{l, s, p, f}(z, t)$ as follows

$$
\begin{gathered}
A_{l, s, p}(z, t)=\left(2 \pi \omega_{l, s, p} /\left(c n_{l, s, p} \cos \theta_{l, s, p}\right)\right)^{1 / 2} B_{l, s, p}(\xi) \exp \left[i \Phi_{l, s, p}(\xi)\right], \\
\tilde{u}_{f}(z, t)=\left(2 \pi \omega_{f} /\left(c n_{p} \cos \theta_{p}\right)\right)^{1 / 2} B_{f}(\xi) \exp \left[i \Phi_{f}(\xi)\right],
\end{gathered}
$$

where the real amplitudes $B_{l, s, p, f}$ and phases $\Phi_{l, s, p, f}$ are considered to be functions of only $\xi=t-z / v, v$ is the soliton velocity; $n_{l, s, p}$ are the indexes of refraction in linear theory. The soliton solution was found to be of Lorentzian shape:

$$
\begin{aligned}
& Q(\xi)=\left(4 \alpha^{2} / \kappa^{2}\right) /\left(1+\alpha^{4} \xi^{2} / \kappa^{2}\right), \\
& \cos \Phi(\xi)=-1 /\left(1+\alpha^{4} \xi^{2} / \kappa^{2}\right),
\end{aligned}
$$

where:

$$
\begin{gathered}
Q(\xi) \equiv B_{l}^{2}(\xi) / \lambda_{l}^{2}=B_{s}^{2}(\xi) / \lambda_{s}^{2}=B_{p}^{2}(\xi) / \lambda_{p}^{2}=B_{f}^{2}(\xi) / \lambda_{f}^{2}, \\
\Phi(\xi) \equiv \Phi_{l}(\xi)-\Phi_{s}(\xi)-\tilde{\Phi}(\xi), \sigma \equiv k_{p} / W, \quad \tilde{\Phi}(\xi)=\Phi_{p}(\xi)=\Phi_{f}(\xi)
\end{gathered}
$$

or

$$
\begin{gathered}
\tilde{\Phi}(\xi)=\Phi_{p}(\xi)=\Phi_{f}(\xi) \pm \pi, \quad \lambda_{l}^{2} \equiv-\kappa_{l} \sigma \chi^{2}\left(\kappa_{p}+J^{2} \eta /\left(\sigma \chi^{2}\right)\right), \quad \chi \equiv m \chi_{0}, \\
\lambda_{f}^{2} \equiv J^{2} \eta^{2}, \quad \lambda_{s}^{2} \equiv \kappa_{s} \sigma \chi^{2}\left(\kappa_{p}+J^{2} \eta /\left(\sigma \chi^{2}\right)\right), \quad \lambda_{p}^{2}=\kappa_{p}^{2} \sigma^{2} \chi^{2} ; \kappa_{l, s, p}=v \cdot v_{l, s, p}^{2} /\left(v-v_{l, s, p}^{2}\right), \\
\alpha \equiv 2 \lambda_{l} \lambda_{s}, \kappa \equiv\left(\kappa_{s} \gamma_{1}-\kappa_{l} \gamma_{2}\right) \lambda_{l}^{2}+\left(\kappa_{s} \gamma_{3}-\kappa_{l} \gamma_{1}\right) \lambda_{s}^{2}, \\
m \equiv\left(8 \pi^{3} \omega_{l} \omega_{s} \omega_{p} /\left(c^{3} n_{l} n_{s} n_{p} \cos \vartheta_{l}^{2} \cos \theta_{s}^{2} \cos \theta_{p}^{2}\right)\right)^{1 / 2}, \quad J \equiv m \eta_{f} \omega_{f}^{2} \alpha^{(f)} / g^{(f)} \\
\eta \equiv g^{(f) 2} c n_{p} \cos \theta_{p} /\left(s_{f} \omega_{f}^{2} \omega_{p}\right), \quad \gamma_{1} \equiv 4 \pi^{2} \gamma_{0} \omega_{l} \omega_{s} /\left(c^{2} n_{l} n_{s} \cos \theta_{l} \cos \theta_{s}\right) \\
\gamma_{2} \equiv 4 \pi^{2} \gamma_{0} \omega_{l}^{2} /\left(c^{2} n_{l}^{2} \cos ^{2} \theta_{l}\right), \quad \gamma_{3} \equiv 4 \pi^{2} \gamma_{0} \omega_{s}^{2} /\left(c^{2} n_{s}^{2} \cos ^{2} \theta_{s}\right) .
\end{gathered}
$$

\section{Finite-Difference Equivalent of Basic System of Equations}

In this paper, we apply the method of finite differences to show that space-time evolution of impulses is consistent with asymptotic behaviour in form of solitons. For the time derivative the forward difference was applied [11]:

$$
\frac{\partial u}{\partial t} \approx \frac{u_{m}^{n+1}-u_{m}^{n}}{\tau} \equiv \frac{u(m, n+1)-u(m, n)}{\tau},
$$

For the space derivative the backward difference was applied:

$$
\frac{\partial u}{\partial z} \approx \frac{u_{m}^{n}-u_{m-1}^{n}}{h} \equiv \frac{u(m, n)-u(m-1, n)}{h},
$$


The system (4)-(7) can be rewritten as follows

$$
\begin{gathered}
\frac{A L(m, n)-A L(m-1, n)}{h}+\frac{1}{V L} \cdot \frac{A L(m, n+1)-A L(m, n)}{\tau}=F L 1(m, n), \\
\frac{P H L(m, n)-P H L(m-1, n)}{h}+\frac{1}{V L} \cdot \frac{P H L(m, n+1)-P H L(m, n)}{\tau}=F L 2(m, n), \\
\frac{A S(m, n)-A S(m-1, n)}{h}+\frac{1}{V S} \cdot \frac{A S(m, n+1)-A S(m, n)}{\tau}=F S 1(m, n), \\
\frac{P H S(m, n)-P H S(m-1, n)}{h}+\frac{1}{V S} \cdot \frac{P H S(m, n+1)-P H S(m, n)}{\tau}=F S 2(m, n), \\
\frac{A P(m, n)-A P(m-1, n)}{h}+\frac{1}{V P} \cdot \frac{A P(m, n+1)-A P(m, n)}{\tau}=F P 1(m, n), \\
\frac{P H P(m, n)-P H P(m-1, n)}{h}+\frac{1}{V P} \cdot \frac{P H P(m, n+1)-P H P(m, n)}{\tau}=F P 2(m, n), \\
\frac{A F(m, n+1)-A F(m, n)}{\tau}=F F 1(m, n), \\
\frac{P H F(m, n+1)-P H F(m, n)}{\tau}=F F 2(m, n),
\end{gathered}
$$

where:

$$
\begin{gathered}
A L \equiv\left|B_{l}(\tilde{z}, \tilde{t})\right|, \quad A S \equiv\left|B_{s}(\tilde{z}, \tilde{t})\right|, \quad A P \equiv\left|B_{p}(\tilde{z}, \tilde{t})\right|, \quad A F \equiv\left|B_{f}(\tilde{t})\right|, \\
P H L \equiv \varphi_{l}(\tilde{z}, \tilde{t}), \quad P H S \equiv \varphi_{s}(\tilde{z}, \tilde{t}), \quad P H F \equiv \varphi_{f}(\tilde{z}, \tilde{t}),
\end{gathered}
$$

$F L 1(m, n) \equiv-(K L 1) A S(m, n) A P(m, n) \sin \varphi_{1}(m, n)-(K L 2) A S(m, n) A F(m, n) \sin \varphi_{2}(m, n)$,

$$
P H P \equiv \varphi_{p}(\tilde{z}, \tilde{t}),
$$

$F L 2(m, n) \equiv(K L 1) \cdot A S(m, n) \cdot A P(m, n) / A L(m, n) \cdot \cos \varphi_{1}(m, n)$

$+(K L 2) \cdot A S(m, n) \cdot A F(m, n) / A L(m, n) \cdot \cos \varphi_{2}(m, n)+(K L 3) \cdot A L^{2}(m, n)+(K L 4) \cdot A S^{2}(m, n)$,

$F S 1(m, n) \equiv(K S 1) A L(m, n) A P(m, n) \sin \varphi_{1}(m, n)+(K S 2) \cdot A L(m, n) \cdot A F(m, n) \cdot \sin \varphi_{2}(m, n)$,

$F S 2(m, n) \equiv(K S 1) \cdot A L(m, n) A P(m, n) / A S(m, n) \cos \varphi_{1}(m, n)$

$+(K S 2) \cdot A L(m, n) \cdot A F(m, n) / A S(m, n) \cdot \cos \varphi_{2}(m, n)+(K S 3) \cdot A L^{2}(m, n)+(K S 4) \cdot A S^{2}(m, n)$,

$F P 1(m, n) \equiv-(K P 2) \cdot A F(m, n) \cdot \sin \varphi_{3}(m, n)+(K P 3) \cdot A L(m, n) \cdot A S(m, n) \cdot \sin \varphi_{1}(m, n)$,

$$
\begin{aligned}
F P 2(m, n) \equiv & -(K P 1)+(K P 2) \cdot A F(m, n) / A P(m, n) \cos \varphi_{3}(m, n) \\
& +(K P 3) \cdot A L(m, n) A S(m, n) / A P(m, n) \cos \varphi_{1}(m, n),
\end{aligned}
$$

$F F 1(m, n) \equiv(K F 2) \cdot A P(m, n) \sin \varphi_{3}(m, n)+(K F 3) \cdot A L(m, n) A S(m, n) \sin \varphi_{2}(m, n)$,

$$
\begin{gathered}
F F 2(m, n) \equiv-(K F 1)+(K F 2) A P(m, n) / A F(m, n) \cdot \cos \varphi_{3}(m, n) \\
+(K F 3) \cdot A L(m, n) \cdot A S(m, n) / A F(m, n) \cdot \cos \varphi_{2}(m, n), \\
\varphi_{1}(m, n) \equiv P H S(m, n)+\operatorname{PHP}(m, n)-P H L(m, n),
\end{gathered}
$$$$
\varphi_{2}(m, n) \equiv P H S(m, n)+\operatorname{PHF}(m, n)-P H L(m, n), \quad \varphi_{3}(m, n) \equiv \operatorname{PHF}(m, n)-P H P(m, n),
$$ 


$$
\begin{gathered}
K L 1 \equiv k l 1 \cdot \tilde{m}, \quad K L 2 \equiv k l 2 \cdot \tilde{m}, \quad K L 3 \equiv k l 3 \cdot \tilde{n}, \quad K L 4 \equiv k l 3 \cdot \tilde{p}, \quad K S 1 \equiv k s 1 \cdot \tilde{m}, \\
K S 2 \equiv k s 2 \cdot \tilde{m}, \quad K S 3 \equiv k s 3 \cdot \tilde{p}, \quad K S 4 \equiv k s 3 \cdot \tilde{q}, \quad K P 1 \equiv \tilde{r}_{p}^{(1)}, \quad K P 2 \equiv \tilde{r}_{p}^{(2)}, \quad K P 3 \equiv(k p 1) \sigma \tilde{m}, \\
\sigma=k_{p} / W, \quad K F 1 \equiv k f 1, \quad K F 2 \equiv k f 2, \quad K F 3 \equiv k f 3 \cdot \tilde{m}, \quad k f 1 \equiv \Delta \omega \tau_{0}, \\
k l 1=k s 1=k p 1=\chi^{\infty} A_{0}, \quad k l 2=k s 2 \equiv \eta_{f} \omega_{f}^{2} \alpha^{(f)} z_{0} / g^{(f)}, \quad k f 2 \equiv g^{(f)} \tau_{0} A_{0} /\left(2 \omega_{f} z_{0}\right), \quad k l 3=k s 3 \equiv \gamma^{\infty} A_{0}^{2}, \\
\tilde{m} \equiv\left(8 \pi^{3} \omega_{l} \omega_{s} \omega_{p} z_{0}^{3} /\left(c^{3} n_{l} n_{s} n_{p} \cos \theta_{l}^{z} \cos \theta_{s}^{z} \cos \theta_{p}^{z}\right)\right)^{1 / 2}, \tilde{n} \equiv\left(2 \pi \omega_{l} z_{0} /\left(c n_{l} \cos \theta_{l}^{z}\right)\right)^{2}, \\
\tilde{p} \equiv 4 \pi^{2} \omega_{l} \omega_{s} z_{0}^{2} /\left(c^{2} n_{l} n_{s} \cos \theta_{l}^{z} \cos \theta_{s}^{z}\right), \quad k f 3 \equiv \eta_{f} \alpha^{(f)} g^{(f)} \tau_{0} A_{0}^{2} c n_{p} \cos \theta_{p}^{z} /\left(s_{f} \omega_{p} \omega_{f} z_{0}^{2}\right), \\
q_{p} \equiv \omega_{p} / c, \quad \tilde{r}_{p}^{(2)} \equiv q_{p}^{2} \varepsilon_{p}^{\infty} z_{0} /\left(2 w^{z}\right)\left(w^{2} /\left(q_{p}^{2} \varepsilon_{p}^{\infty}\right)-1\right), \quad \tilde{q} \equiv\left(2 \pi \omega_{s} z_{0} /\left(c n_{s} \cos \theta_{s}^{z}\right)\right)^{2}, \\
\tilde{r}_{p}^{(1)} \equiv s_{f} \omega_{f}^{2} \omega_{p}^{2} z_{0}^{2} /\left(2 g^{(f)} w^{z} c^{2} A_{0}\right), \quad B_{l, s, p, f}(\tilde{z}, \tilde{t}) \equiv\left|B_{l, s, p, f}(\tilde{z} . \tilde{t})\right| \exp \left\{i \varphi_{l, s, p, f}(\tilde{z}, \tilde{t})\right\}, \quad V L \equiv \tilde{v}_{l}^{z}, V S \equiv \tilde{v}_{s}^{z}, V P \equiv \tilde{v}_{p}^{z}
\end{gathered}
$$

The chosen mesh was

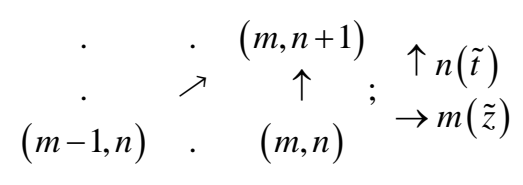

\section{Transition Regime of the Stimulated Raman Scattering Solitons}

The pulses on laser and Stokes frequencies were chosen to be of Gaussian shape (their amplitudes were proportional to $\exp \left(-\alpha \tilde{t}^{2}\right)$ (the coefficient $\alpha$ was 0.159155 ; this was done in order to minimize the transition regime and to get the area of each pulse close to $\pi$ ). The two-dimensional arrays $A$ for the amplitudes of waves were $A$ [10] [1000] which resulted in 10,000 points for each wave. The conservation of energy (in our case the area under the pulse shape) was monitored by calculating the time integrals for each cross-sectional area in a medium. Since we suggested that the normalized areas of incoming waves (area divided by the amplitude) were equal to $\pi$, all those integrals showed the evolution of pulses of Gaussian shape to those of Lorentzian shape with normalized area of $\pi\left(\tau_{0} \approx 10^{-15} \mathrm{~s}, A_{0} \approx 10^{8} \mathrm{esu}, \chi^{\infty} \approx 10^{-8} \mathrm{esu}, \gamma^{\infty} \approx 10^{-15}\right.$ esu [13]). The results are shown in Figure 1.

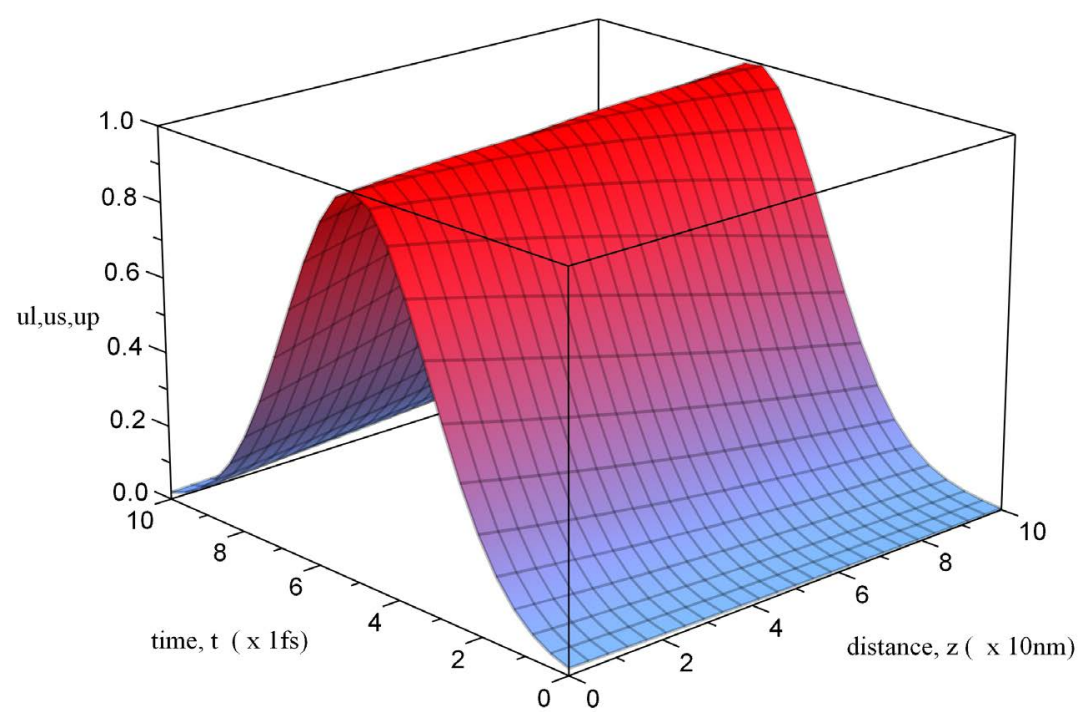

Figure 1. The space-time evolution of the normalized intensities $\left(u l, u s, u p \equiv B_{l, s, p}^{2}(\tilde{z}, \tilde{t}) / B_{l, s, p, \max }^{2}(\tilde{z})\right)$ of laser, Stokes, and polariton pulses. 


\section{Stability of the Stimulated Raman Scattering Solitons}

Let the new amplitudes (amplitudes with disturbances) in unitless form be

$$
\begin{array}{r}
B_{l, s, p}^{\prime}(\tilde{z}, \tilde{t})=\tilde{B}_{l, s, p}(\tilde{\xi}) \exp \left[i \cdot \Phi_{l, s, p}(\tilde{\xi})\right]+\tilde{\Psi}_{l, s, p}(\tilde{t}), \\
B_{f}^{\prime}(\tilde{z}, \tilde{t})=\tilde{B}_{f}(\tilde{\xi}) \exp \left[i \cdot \Phi_{f}(\tilde{\xi})\right]+\tilde{\Psi}_{f}(\tilde{t}),
\end{array}
$$

where: $B_{l, s, p, f}^{\prime}(\tilde{z}, \tilde{t})$ are the unitless amplitudes of waves in presence of disturbance $\left(B_{l, s, p, f}^{\prime}=\left(B_{l, s, p, f} / A_{0}\right)\right)$; $\tilde{\Psi}_{l, s, p, f}=\Psi_{l, s, p, f} / A_{0}$ are the small disturbances; $A_{0}$-is the peak amplitude of the incoming laser pulse; $\tilde{\xi}=\xi / \tau_{0} ; \tilde{t}=t / \tau_{0} ; \tilde{z}=z / z_{0} ; z_{0}=c \cdot \tau_{0} ; c$ is the speed of light in vacuum; $\tau_{0}$ is the characteristic time $\left(\tau_{0}=\right.$ $1 \mathrm{fs}$ ). When we substitute (22) and (23) into the system (4)-(7) we get the system of equations that can be significantly simplified if we consider the evolution of disturbances $\tilde{\Psi}_{l, s, p, f}$ in the vicinity of the maximum value of soliton amplitude that is in the case when $\tilde{\xi}=0$. In that case the simplified system of equations (the disturbances $\tilde{\Psi}_{l, s, p, f}$ are considered to be small compared to the amplitudes of solitons) becomes that of four linear equations of second order:

$$
\frac{1}{\tilde{v}_{l}^{2}} \frac{\partial^{2}}{\partial \tilde{t}^{2}} \operatorname{Re}\left\{\tilde{\Psi}_{l}(\tilde{t})\right\}=(A 1) \operatorname{Re}\left\{\tilde{\Psi}_{l}(\tilde{t})\right\}+(A 2) \operatorname{Re}\left\{\tilde{\Psi}_{s}(\tilde{t})\right\}+(A 3) \operatorname{Re}\left\{\tilde{\Psi}_{p}(\tilde{t})\right\}+(A 4) \operatorname{Re}\left\{\tilde{\Psi}_{f}(\tilde{t})\right\},
$$

where:

$$
\begin{aligned}
& A 1 \equiv \tilde{v}_{l}^{z} \cdot \operatorname{Re}^{2}\{L L 1(0)\}-\tilde{v}_{l}^{z} \cdot \operatorname{Re}^{2}\{L L 2(0)\}+\tilde{v}_{s}^{z} \cdot \operatorname{Re}\{S L 1(0)\}(\operatorname{Re}\{L S 1(0)\}-\operatorname{Re}\{L S 2(0)\}) \\
& +\tilde{v}_{s}^{z} \cdot \operatorname{Re}\{S L 2(0)\} \cdot(\operatorname{Re}\{L S 1(0)\}-\operatorname{Re}\{L S 2(0)\}) \\
& -\tilde{v}_{p}^{z} \cdot \operatorname{Re}\{L P 1(0)\} \cdot \operatorname{Re}\{P L 1\{0\}\}-\operatorname{Re}\{L F 1(0)\} \cdot \operatorname{Re}\{F L 1(0)\} \text {, } \\
& A 2 \equiv \tilde{v}_{l}^{2} \cdot(\operatorname{Re}\{L L 1(0)\}-\operatorname{Re}\{L L 2(0)\}) \cdot(\operatorname{Re}\{L S 1(0)\}+\operatorname{Re}\{L S 2(0)\}) \\
& +\tilde{v}_{s}^{2} \cdot(\operatorname{Re}\{L S 1(0)\}-\operatorname{Re}\{L S 2(0)\}) \cdot(\operatorname{Re}\{S S 1(0)\}+\operatorname{Re}\{S S 2(0)\}) \\
& -\tilde{v}_{p}^{z} \cdot \operatorname{Re}\{L P 1(0)\} \cdot \operatorname{Re}\{P S 1(0)\}-\operatorname{Re}\{L F 1(0)\} \cdot \operatorname{Re}\{F S 1(0)\}, \\
& A 3 \equiv \tilde{v}_{l}^{z} \cdot \operatorname{Re}\{L P 1(0)\}(\operatorname{Re}\{L L 1(0)\}-\operatorname{Re}\{L L 2(0)\})+\tilde{v}_{s}^{z} \operatorname{Re}\{S P 1(0)\}(\operatorname{Re}\{L S 1(0)\}-\operatorname{Re}\{L S 2(0)\}) \\
& +\tilde{v}_{p}^{z} \operatorname{Re}\{L P 1(0)\} \cdot \operatorname{Re}\{P P 1(0)\}-\operatorname{Re}\{L F 1(0)\} \cdot \operatorname{Re}\{F P 1(0)\}, \\
& A 4 \equiv \tilde{v}_{l}^{z} \cdot \operatorname{Re}\{L F 1(0)\} \cdot(\operatorname{Re}\{L L 1(0)\}-\operatorname{Re}\{L L 2(0)\})+\tilde{v}_{s}^{2} \cdot \operatorname{Re}\{S F 1(0)\}(\operatorname{Re}\{L S 1(0)\}-\operatorname{Re}\{L S 2(0)\}) \\
& -\tilde{v}_{p}^{z} \operatorname{Re}\{L P 1(0)\} \operatorname{Re}\{P F 1(0)\}+\operatorname{Re}\{L F 1(0)\} \operatorname{Re}\{F F 1(0)\}, \\
& L L 1(0) \equiv(k l 3) \cdot \tilde{n} \cdot \tilde{B}_{l}^{2}(0), \quad L L 2(0) \equiv(k l 3)\left(2 \tilde{n} \tilde{B}_{l}^{2}(0)+\tilde{p}_{s}^{2}(0)\right), \quad L S 1(0) \equiv(k l 3) \cdot \tilde{p} \cdot \tilde{B}_{l}(0) \cdot \tilde{B}_{s}(0), \\
& L S 2(0) \equiv(k l 1) \tilde{m} \tilde{B}_{p}(0)+(k l 2) \tilde{m} \tilde{B}_{f}(0)+(k l 3) \tilde{p} \tilde{B}_{l}(0) \tilde{B}_{s}(0), \quad S L 1(0) \equiv(k l 3) \tilde{p} \tilde{B}_{l}(0) \tilde{B}_{s}(0), \\
& L P 1(0) \equiv(k l 1) \cdot \tilde{m} \cdot \tilde{B}_{s}(0), \quad S L 2(0) \equiv(k s 1) \tilde{m} \tilde{B}_{p}(0)+(k s 2) \tilde{m} \tilde{B}_{f}(0)+(k s 3) \tilde{p} \tilde{B}_{l}(0) \tilde{B}_{s}(0), \\
& L F 1(0) \equiv(k l 2) \tilde{m} \tilde{B}_{s}(0), \quad P L 1(0) \equiv(k p 3) \tilde{B}_{s}(0), \quad F L 1(0) \equiv(k f 3) \tilde{B}_{s}(0), \quad S S 1(0) \equiv(k s 3) \tilde{q} \tilde{B}_{s}^{2}(0), \\
& S S 2(0) \equiv(k s 3) \tilde{p} \tilde{B}_{l}^{2}(0)+(k s 3) \tilde{q} 2 \tilde{B}_{s}^{2}(0), \quad P S 1(0) \equiv(k p 3) \tilde{B}_{l}(0), \quad F S 1(0) \equiv(k f 3) \tilde{B}_{l}(0), \\
& S P 1(0) \equiv(k s 1) \tilde{m} \tilde{B}_{l}(0), \quad P P 1(0) \equiv(k p 1), \quad F P 1(0) \equiv(k f 2), \\
& S F 1(0) \equiv(k s 2) \tilde{m} \tilde{B}_{l}(0), \quad P F 1(0) \equiv(k p 2), \quad F F 1 \equiv(k f 1), \\
& \frac{1}{\tilde{v}_{s}^{z}} \frac{\partial^{2}}{\partial \tilde{t}^{2}} \operatorname{Re}\left\{\tilde{\Psi}_{s}(\tilde{t})\right\}=(B 1) \operatorname{Re}\left\{\tilde{\Psi}_{l}(\tilde{t})\right\}+(B 2) \operatorname{Re}\left\{\tilde{\Psi}_{s}(\tilde{t})\right\}+(B 3) \operatorname{Re}\left\{\tilde{\Psi}_{p}(\tilde{t})\right\}+(B 4) \operatorname{Re}\left\{\tilde{\Psi}_{f}(\tilde{t})\right\},
\end{aligned}
$$

where: 


$$
\begin{gathered}
B 1 \equiv \tilde{v}_{l}^{z}(\operatorname{Re}\{S L 1(0)\}-\operatorname{Re}(S L 2(0))) \cdot(\operatorname{Re}\{L L 1(0)\}+\operatorname{Re}(L L 2(0)))+\tilde{v}_{s}^{z}(\operatorname{Re}\{S S 1\}(0)) \\
-\operatorname{Re}\{S S 2(0)\} \cdot(\operatorname{Re}\{S L 1(0)\}+\operatorname{Re}\{S L 2(0)\})+\tilde{v}_{p}^{z} \operatorname{Re}\{S P 1(0)\} \operatorname{Re}\{P L 1(0)\}+\operatorname{Re}\{S F 1(0)\} \operatorname{Re}\{F L 1(0)\}, \\
B 2 \equiv \tilde{v}_{l}^{z}(\operatorname{Re}\{S L 1(0)\}-\operatorname{Re}\{S L 2(0)\})(\operatorname{Re}\{L S 1(0)\}+\operatorname{Re}\{L S 2(0)\})+\tilde{v}_{s}^{z}\left(\operatorname{Re}^{2}\{S S 1(0)\}-\operatorname{Re}^{2}\{S S 2\{0\}\}\right), \\
B 3 \equiv \tilde{v}_{l}^{z} \operatorname{Re}\{L P 1(0)\}(\operatorname{Re}\{S L 1(0)\}-\operatorname{Re}\{S L 2(0)\})+\tilde{v}_{s}^{z} \operatorname{Re}\{S P 1(0)\}(\operatorname{Re}\{S S 1\}(0)-\operatorname{Re}\{S S 2(0)\}) \\
\quad-\tilde{v}_{p}^{z} \operatorname{Re}\{S P 1(0)\} \operatorname{Re}\{P P 1(0)\}+\operatorname{Re}\{S F 1\{0\}\} \operatorname{Re}\{F P 1(0)\}, \\
B 4 \equiv \tilde{v}_{l}^{z} \operatorname{Re}\{L F 1(0)\} \operatorname{Re}\{S L 1(0)\}-\tilde{v}_{l}^{z} \cdot \operatorname{Re}\{L F 1(0)\} \operatorname{Re}\{S L 2(0)\}+\tilde{v}_{s}^{z} \operatorname{Re}\{S F 1(0)\}(\operatorname{Re}\{S S 1(0)\}-\operatorname{Re}\{S S 2(0)\}) \\
+\tilde{v}_{p}^{z} \operatorname{Re}\{S P 1(0)\} \operatorname{Re}\{P F 1(0)\}-\operatorname{Re}\{S F 1(0)\} \operatorname{Re}\{F F 1(0)\}, \\
\frac{1}{\tilde{v}_{p}^{z}} \frac{\partial^{2}}{\partial \tilde{t}^{2}} \operatorname{Re}\left\{\tilde{\Psi}_{p}(\tilde{t})\right\}=(C 1) \operatorname{Re}\left\{\tilde{\Psi}_{l}(\tilde{t})\right\}+(C 2) \operatorname{Re}\left\{\tilde{\Psi}_{s}(\tilde{t})\right\}+(C 3) \operatorname{Re}\left\{\tilde{\Psi}_{p}(\tilde{t})\right\}+(C 4) \operatorname{Re}\left\{\tilde{\Psi}_{f}(\tilde{t})\right\},
\end{gathered}
$$

where:

$$
\begin{gathered}
C 1 \equiv-\tilde{v}_{l}^{z} \operatorname{Re}\{P L 1(0)\}(\operatorname{Re}\{L L 1(0)\}+\operatorname{Re}\{L L 2(0)\})+\tilde{v}_{s}^{z} \operatorname{Re}\{P S 1(0)\} \operatorname{Re}\{S L 1(0)\} \\
+\tilde{v}_{s}^{z} \operatorname{Re}\{P S 1(0)\} \cdot \operatorname{Re}\{S L 2(0)\}+\tilde{v}_{p}^{z} \operatorname{Re}\{P P 1(0)\} \operatorname{Re}\{P L 1(0)\}-\operatorname{Re}\{P F 1(0)\} \operatorname{Re}\{F L 1(0)\} \\
C 2 \equiv-\tilde{v}_{l}^{z} \operatorname{Re}\{P L 1(0)\} \operatorname{Re}\{L S 1(0)\}-\tilde{v}_{l}^{z} \operatorname{Re}\{P L 1(0)\} \operatorname{Re}\{L S 2(0)\} \\
+\tilde{v}_{s}^{z} \operatorname{Re}\{P S 1(0)\}(\operatorname{Re}\{S S 1(0)\}+\operatorname{Re}\{S S 2(0)\}) \\
+\tilde{v}_{p}^{z} \operatorname{Re}\{P P 1(0)\} \operatorname{Re}\{P S 1(0)\}-\operatorname{Re}\{P F 1(0)\} \operatorname{Re}\{F S 1(0)\} \\
C 3 \equiv-\tilde{v}_{l}^{z} \operatorname{Re}\{P L 1(0)\} \operatorname{Re}\{L P 1(0)\}+\tilde{v}_{s}^{z} \operatorname{Re}\{P S 1(0)\} \operatorname{Re}\{S P 1(0)\} \\
\quad-\tilde{v}_{p}^{z} \operatorname{Re}\{P P 1(0)\} \operatorname{Re}\{P P 1(0)\}-\operatorname{Re}\{P F 1(0)\} \operatorname{Re}\{F P 1(0)\}, \\
C 4 \equiv-\tilde{v}_{l}^{z} \cdot \operatorname{Re}\{P L 1(0)\} \cdot \operatorname{Re}\{L F 1(0)\}+\tilde{v}_{s}^{z} \cdot \operatorname{Re}\{P S 1(0)\} \cdot \operatorname{Re}\{S F 1(0)\} \\
+\tilde{v}_{p}^{z} \operatorname{Re}\{P P 1(0)\} \operatorname{Re}\{P F 1(0)\}+\operatorname{Re}\{P F 1(0)\} \operatorname{Re}\{F F 1(0)\}, \\
\frac{\partial^{2}}{\partial \tilde{t}^{2}} \operatorname{Re}\left\{\tilde{\Psi}_{f}(\tilde{t})\right\}=(D 1) \operatorname{Re}\left\{\tilde{\Psi}_{l}(\tilde{t})\right\}+(D 2) \operatorname{Re}\left\{\tilde{\Psi}_{s}(\tilde{t})\right\}+(D 3) \operatorname{Re}\left\{\tilde{\Psi}_{p}(\tilde{t})\right\}+(D 4) \operatorname{Re}\left\{\tilde{\Psi}_{f}(\tilde{t})\right\},
\end{gathered}
$$

where:

$$
\begin{aligned}
& D 1 \equiv-\tilde{v}_{l}^{z} \operatorname{Re}\{F L 1(0)\}(\operatorname{Re}\{L L 1(0)\}+\operatorname{Re}\{L L 2(0)\})+\tilde{v}_{s}^{z} \operatorname{Re}\{F S 1\{0\}\}(\operatorname{Re}\{S L 1(0)\}+\operatorname{Re}\{S L 2(0)\}) \\
&-\tilde{v}_{p}^{z} \operatorname{Re}\{F P 1(0)\} \operatorname{Re}\{P L 1(0)\}+\operatorname{Re}\{F F 1(0)\} \operatorname{Re}\{F L 1(0)\}, \\
& D 2 \equiv-\tilde{v}_{l}^{z} \operatorname{Re}\{F L 1(0)\}(\operatorname{Re}\{L S 1(0)\}+\operatorname{Re}\{L S 2(0)\})+\tilde{v}_{s}^{z} \operatorname{Re}\{F S 1(0)\}(\operatorname{Re}\{S S 1(0)\}+\operatorname{Re}\{S S 2(0)\}) \\
&-\tilde{v}_{p}^{z} \operatorname{Re}\{F P 1(0)\} \operatorname{Re}\{P S 1(0)\}-\operatorname{Re}\{F F 1(0)\} \cdot \operatorname{Re}\{F S 1(0)\}, \\
& D 3 \equiv--\tilde{v}_{l}^{z} \operatorname{Re}\{F L 1(0)\} \operatorname{Re}\{L P 1(0)\}+\tilde{v}_{s}^{z} \operatorname{Re}\{F S 1(0)\} \operatorname{Re}\{S P 1(0)\}+\tilde{v}_{p}^{z} \operatorname{Re}\{F P 1(0)\} \operatorname{Re}\{P P 1(0)\} \\
&+\operatorname{Re}\{F F 1(0)\} \cdot \operatorname{Re}\{F P 1(0)\}, \\
& D 4 \equiv-\tilde{v}_{l}^{z} \operatorname{Re}^{2}\{F L 1(0)\}+\tilde{v}_{s}^{z} \operatorname{Re}(F S 1(0)) \operatorname{Re}\{S F 1(0)\}-\tilde{v}_{p}^{z} \operatorname{Re}\{F P 1(0)\} \operatorname{Re}\{P P 1(0)\}-\operatorname{Re}^{2}\{F F 1(0)\}, \\
& \tilde{v}_{l, s, p}^{z} \equiv 1 /\left(n_{l, s, p} \cos \theta_{l, s, p}^{z}\right) .
\end{aligned}
$$

The system of equations (24)-(27) was solved numerically. The results are presented in Figure 2. 


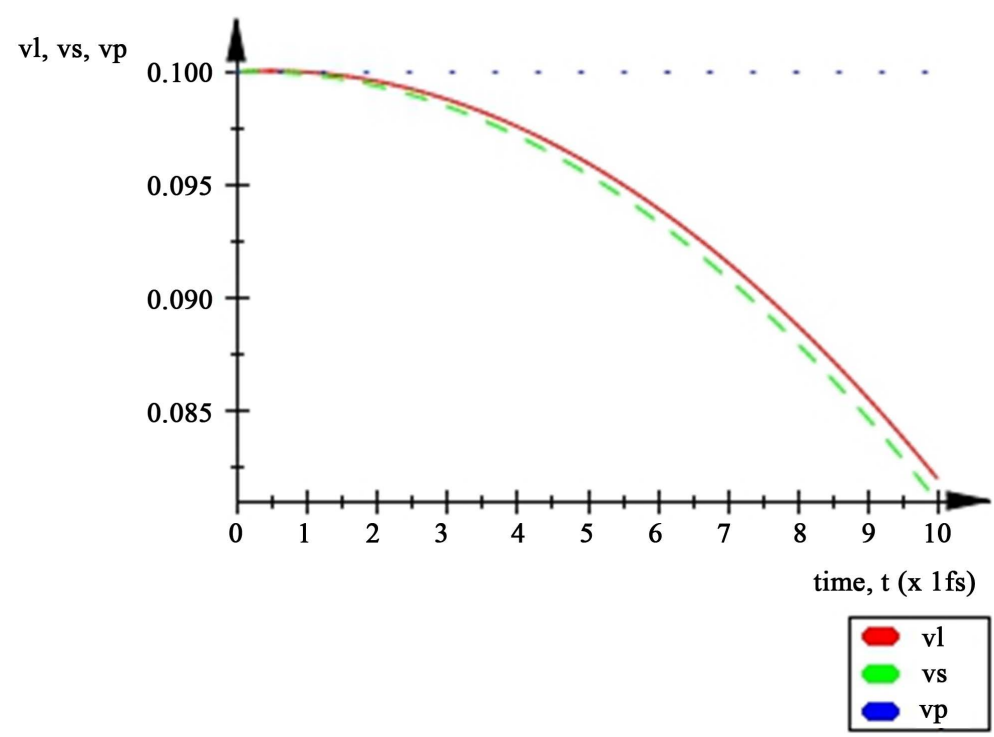

Figure 2. The time evolution of normalized disturbances $\left(v l \equiv \tilde{\psi}_{l}(t)\right.$, red solid line; $v s \equiv \tilde{\psi}_{s}(t)$, green dashed line, $\quad v p \equiv \tilde{\psi}_{p}(t)$, blue dotted line) at $A 1=A 2=-18$, $A 3=A 4=-4, \quad B 1=B 2=-14, \quad B 3=B 4=-4, \quad C 1=0, \quad C 2=0, \quad C 3=-2, \quad C 4=2$, $D 1=D 2=0, \quad D 3=2, \quad D 4=-2$.

\section{Conclusion}

The system of eight nonlinear equations describing the SRS with excitation of polar optical phonons was numerically solved. The results of the computer simulation are completely consistent with the asymptotical solutions in form of solitons. It is numerically shown that with SRS on dipole-active phonons the process of propagation of pulses of Gaussian shape on laser and Stokes frequencies results in formation of solitons of Lorentzian shape at all frequencies of interacting waves. Those solitons are stable in wide range of parameters that characterize both the electromagnetic waves and medium.

\section{References}

[1] Rolle, J., Berge, L., Duchateau, G. and Skupin, S. (2014) Filamentation of Ultrashort Laser Pulses in Silica Glass and KDP Crystal: A Comparative Study. Physical Review A, 90, 1-8. http://dx.doi.org/10.1103/PhysRevA.90.023834

[2] Chen, Z., Segev, M. and Christodoulides, D.N. (2012) Optical Spatial Solitons: Historical Overview and Recent Advances. Reports on Progress in Physics, 75, 1-20. http://dx.doi.org/10.1088/0034-4885/75/8/086401

[3] Prokopovich, I.P. and Khrushchinskii, A.A. (1997) Highly Efficient Generation of Attosecond Pulses in Coherent Stimulated Raman Self-Scattering of Intense Femtosecond Laser Pulses. Laser Physics, 7, 305-308.

[4] Prokopovich, I.P. and Peatross, J. (1999) Direct Shaping and Amplifying of High-Intense Single Attosecond Pulses from High-Intense Femtosecond Optical Pulses in Inert Gases. Laser Physics, 9, 588-591.

[5] Kaplan, A.E. and Shkolnikov, P.L. (1996) Subfemtosecond Pulses in the Multicascade Stimulated Raman Scattering. Journal of the Optical Society of America B, 13, 347-354. http://dx.doi.org/10.1364/JOSAB.13.000347

[6] Makhviladze, T.M. and Sarychev, M.E. (1976) Soliton Regimes of Stimulated Raman Scattering. Soviet PhysicsJETP, 44, 471-477.

[7] Makhviladze, T.M., Sarychev, M.E. and Shelepin L.A. (1975) Raman Scattering of Light by an Excited Medium. Soviet Physics-JETP, 42, 255-261.

[8] Kivshar, Y. and Agrawal, G. (2003) Optical Solitons: From Fibers to Photonic Crystals. Academic Press, Waltham.

[9] Ames, W. (1992) Numerical Methods for Partial Differential Equations. 3rd Edition, Academic Press, Waltham.

[10] Farlow, S. (1993) Partial Differential Equations for Scientists and Engineers. Dover Books, Mineola.

[11] Kreyszig, E. (1993) Advanced Engineering Mathematics. 7th Edition, John Wiley \& Sons Ltd., Hoboken. 
[12] Marchevskii, F.N., Strizhevskii, V.L. and Feshchenko, V.P. (1984) Soliton Generation by Stimulated Raman Scattering with Excitation of Polar Optical Phonons. Quantum Electronics, 11, 277-282. http://dx.doi.org/10.1070/qe1984v014n02abeh004691

[13] Boyd, R. (2008) Nonlinear Optics. 3rd Edition, Academic Press, Waltham. 